\section{Hipertensão arterial sistêmica auto-referida: validação diagnóstica em estudo de base populacional}

\author{
Self-reported hypertension: validation in a \\ representative cross-sectional survey
}

\begin{abstract}
To assess the validity of self-reported hypertension, a population-based cross-sectional study was carried out in Pelotas, Southern Brazil. Individuals aged $\geq 20$ years were selected through probabilistic two-stage sampling. All 2,949 participants had their blood pressure measured twice (5 minutes apart) during a household visit. Those presenting systolic pressure $\geq 140 \mathrm{mmHg}$ and/or diastolic pressure $\geq 90 \mathrm{mmHg}$ were revisited. The gold standard for hypertension was the mean of the two measures taken at the second visit and/or use of anti-hypertensive drugs. Selfreported hypertension was obtained through the question: "Has any doctor ever said that you have high blood pressure?". Prevalence rates for selfreported and measured hypertension were $33.6 \%$ and $29.5 \%$, respectively. Sensitivity was $84.3 \%$ (81.7-86.7\%), specificity 87.5\% (86.0-88.9\%), positive predictive value $73.9 \%$ (71.0-76.6\%), and negative predictive value 93.0\% (91.8-94.1\%). Self-reporting is a reliable method for monitoring prevalence of hypertension, one of the most important risk factors for chronic non-communicable diseases.
\end{abstract}

Hypertension; Chronic Disease; Adult Health
Maria Aurora Dropa Chrestani 1

Iná da Silva dos Santos 1

Alícia M. Matijasevich 1

Nas últimas décadas, as doenças crônicas não transmissíveis têm se destacado como as principais causas de morbimortalidade em todo o mundo. Entre elas, estão as doenças cardiovasculares, com altas prevalências em diferentes populações e em todos os níveis sócio-econômi$\cos { }^{1}$. No Brasil, em 2005, a taxa de mortalidade específica para doença isquêmica do coração foi de 46,1/ 100 mil habitantes e, para doenças cerebrovasculares, de 48,9/100 mil habitantes (Departamento de Informática do SUS. http://tab net.datasus.gov.br/cgi/tabcgi.exe?idb2007/c08. def, acessado em 28/Ago/2008), ambas superiores a todas as demais causas de óbitos no país.

As doenças cardiovasculares têm sido alvo de inúmeros estudos epidemiológicos, o que possibilitou identificar diversos fatores de risco, entre eles a hipertensão arterial sistêmica 2. Estima-se que esta esteja associada a $47 \%$ dos casos de doença isquêmica cardíaca e a $54 \%$ dos casos de acidente vascular cerebral 2 .

As avaliações de impacto de intervenções em larga escala, visando à prevenção primária das doenças crônicas não transmissíveis, incluem a realização periódica de inquéritos populacionais para monitorar a prevalência de seus principais fatores de risco ${ }^{3}$. Nesses inquéritos, a prevalência de hipertensão arterial tem sido obtida através da aferição da pressão arterial ou do auto-relato $4,5,6,7,8,9,10,11,12,13$. O primeiro método, embora 
mais acurado, implica maiores custos e maior complexidade, uma vez que, além de envolver o uso de equipamentos, poderá exigir a revisita dos participantes, para atender os atuais critérios diagnósticos 14. A aplicação de questionários, buscando a informação auto-referida, é uma estratégia de menor custo, acessível e rápida para estimar a prevalência de hipertensão arterial. No entanto, está sujeita a erros de classificação. A acurácia da informação coletada por auto-relato depende de características do questionário e da população à qual ele é aplicado.

O objetivo deste estudo foi avaliar a sensibilidade, especificidade e os valores preditivos positivo e negativo do auto-relato de hipertensão arterial conforme características sócio-demográficas, de comportamento e de utilização de serviços de saúde, em relação ao padrão-ouro, em um estudo de base populacional na cidade de Pelotas, Rio Grande do Sul, Brasil.

\section{Metodologia}

Este estudo faz parte do sistema de consórcio do Programa de Pós-Graduação em Epidemiologia (PPGE) da Universidade Federal de Pelotas (UFPEL). Nesse sistema, todos os mestrandos do PPGE conduzem um único trabalho de campo, no qual são atendidos os objetivos específicos de pesquisa de cada aluno 15 .

Entre os meses de outubro de 2007 e janeiro de 2008, foi realizado um estudo transversal de base populacional entre indivíduos com 20 anos ou mais de idade, residentes na zona urbana do Município de Pelotas. Com cerca de 350 mil habitantes, Pelotas é o terceiro município mais populoso do estado, e 92\% dessa população residem em área urbana (Instituto Brasileiro de Geografia e Estatística. http://www.ibge.gov.br/cidadesat/ topwindow.htm?1, acessado em 01/Set/2007).

\section{Tamanho da amostra}

Para detectar uma sensibilidade maior ou igual a $70 \% 16$ para a hipertensão arterial auto-referida, com erro de 2 p.p., acrescentando $10 \%$ para perdas ou recusas, seriam necessários 1.492 indivíduos hipertensos conforme o padrão-ouro. Para detectar uma especificidade de $86 \% 16$, com erro de 2 p.p. e acrescentando $10 \%$ para perdas ou recusas, seriam necessários 1.430 indivíduos normotensos (conforme o padrão-ouro). Considerando que o presente consórcio deveria incluir aproximadamente 3.500 indivíduos, e que a prevalência de hipertensão arterial em Pelotas estaria em torno de $40 \% 13$, seriam encontrados cerca de 1.400 hipertensos.

\section{Amostragem}

O processo de amostragem foi probabilístico, por conglomerados e em dois estágios. Segundo o censo realizado no ano de 2000 pelo Instituto Brasileiro de Geografia e Estatística (IBGE), a cidade de Pelotas possui 404 setores censitários com domicílios. Com base na quantidade de recursos e tempo disponíveis para a coleta de dados, optouse pela inclusão de 126 setores e 11 domicílios por setor para compor a amostra. Para a seleção dos setores, procedeu-se da seguinte forma: os setores foram ordenados de forma crescente de acordo com a renda média do chefe da família, definidos pelo IBGE. Para a seleção dos domicílios, todos foram, dentro de cada setor, mapeados e numerados no sentido horário. O ponto inicial era indicado sempre no canto inferior direito da área do mapa disponibilizado pelo IBGE. Para saber quais seriam visitados, foi necessário calcular um pulo intra-setor, o que foi feito dividindo-se o número total de domicílios de cada setor por 11 . O primeiro deles foi escolhido de forma aleatória entre o número um e o número total de domicílios para cada setor. Os domicílios subseqüentes foram escolhidos somando-se o valor do pulo ao inicialmente sorteado.

Foram incluídos neste estudo todos os moradores com idade igual ou superior a 20 anos, excluídas as mulheres que sabiam estarem grávidas. Foram classificados como perdas ou recusas aqueles indivíduos que, mesmo após três tentativas de contato, em dias, horários e por entrevistadores diferentes, incluindo a coordenadora deste estudo, não foi possível entrevistar nem obter as medidas de sua pressão arterial.

\section{Variável teste e padrão-ouro}

A variável teste deste estudo foi hipertensão arterial auto-referida, obtida por meio da pergunta: “Algum médico disse que o(a) Sr.(a) tem pressão alta?".

Para definição da hipertensão arterial conforme o padrão-ouro, utilizaram-se dois critérios: (1) o uso de medicação anti-hipertensiva e (2) aferição da pressão arterial. Para os que se disseram hipertensos, investigou-se sobre a prescrição médica de tratamento anti-hipertensivo. $\mathrm{O}$ grupo farmacológico do medicamento foi obtido mediante a revisão de receitas e/ou pela verificação da embalagem de todos os medicamentos prescritos por médico. Posteriormente, a lista de medicamentos de cada entrevistado era revisada pelo supervisor do estudo. Indivíduos com prescrição de anti-hipertensivos, independente de estarem usando ou não algum medicamento, foram classificados como hipertensos. No caso 
de os medicamentos revisados não corresponderem a um dos grupos farmacológicos de drogas anti-hipertensivas (beta-bloqueadores, diuréticos, bloqueador do receptor de aldosterona, inibidores da enzima conversora de angiotensina, antagonistas dos canais de cálcio, bloqueadores da angiotensina, bloqueadores de alfa- 1 , agonistas de alfa-2, vasodilatadores diretos e Guanabenzeno) 17, a classificação como normotenso ou hipertenso era dependente do resultado da medida da pressão arterial.

Independente do auto-relato de ser portador de hipertensão arterial, a pressão arterial foi medida em todos os participantes, utilizando-se o monitor de pulso marca Omron, modelo HEM 629/631 (British Hypertension Society. http:// www.bhsoc.org/bp_monitors/automatic_wrist. stm, acessado em 28/Set/2008. Agência Nacional de Vigilância Sanitária. http://www.anvisa. gov.br/legis/resol/2002/820_02re.htm, acessado em 15/Ago/2007). Todos os monitores de pulso foram inicialmente testados com esfigmomanômetro do tipo aneróide. A primeira medida foi efetuada depois de decorridos os primeiros 15 minutos de entrevista, e a segunda, pelo menos cinco minutos após a primeira. Para a medida da pressão arterial, os indivíduos foram instruídos a ficar sentados durante a entrevista e não deviam estar com vontade de urinar. Além disso, não poderiam ter ingerido qualquer bebida ou alimento, nem fumado, pelo menos nos 30 minutos anteriores à aferição da pressão. Ao final, calculouse a média aritmética das duas medidas. Aqueles que estivessem com pressão arterial inferior a 140/90mmHg, sem uso de anti-hipertensivos, eram classificados como normotensos.

Os indivíduos com pressão arterial sistólica (PAS) $\geq 140 \mathrm{mmHg}$ e/ou pressão arterial diastólica (PAD) $\geq 90 \mathrm{mmHg}$, que não estivessem sob uso de medicação anti-hipertensiva, foram revisitados para uma nova aferição da pressão arterial. Os mesmos cuidados tomados para a medida da pressão na primeira visita foram repetidos na segunda. O tempo médio entre a primeira e a segunda visita foi de 6 semanas \pm 4 semanas.

Quando, na segunda visita, a PAS média das duas medidas da pressão arterial (no início e no final da entrevista) foi igual ou superior a $140 \mathrm{mmHg}$ e/ou a PAD média igual ou superior a 90mmHg, o indivíduo foi classificado como hipertenso (de acordo com os critérios do VII Joint Committee on Detection, Evaluation and Treatment of High Blood Pressure) 14,18.

Todos os indivíduos que não faziam uso de medicamento anti-hipertensivo e que, na primeira visita, apresentaram PAS $<140 \mathrm{mmHg}$ e $\mathrm{PAD}<90 \mathrm{mmHg}$ foram classificados como normotensos (Figura1).

\section{Coleta de dados}

A coleta de dados foi feita no domicílio, mediante a aplicação de dois questionários. Em um, foram obtidas informações sobre as características sócio-demográficas, comportamentais, hipertensão arterial sistêmica, medida da pressão arterial e utilização de serviço médico, para todos os indivíduos entrevistados. No outro questionário, respondido apenas pelo indivíduo que se considerava o chefe da família, obteve-se a informação da renda familiar mensal, que era a soma do valor recebido, em reais, no último mês, para cada morador do domicílio. Posteriormente, este dado foi categorizado em quartis, sendo o primeiro quartil o de menor renda. As informações sócio-demográficas referiam-se a escolaridade, em anos completos de estudo, sexo, idade e cor da pele auto-referida; as comportamentais referiam-se ao tabagismo, sendo considerado tabagista o indivíduo que tivesse fumado um ou mais cigarros no último mês, e ex-tabagista, aquele que não fumava há pelo menos um mês. Quanto à atividade física, foi considerado ativo o indivíduo que praticava atividade física maior ou igual a 150 minutos/semana, segundo o Questionário Internacional de Atividade Física (IPAQ) 19. Para as informações sobre hipertensão arterial, a história familiar de hipertensão arterial do pai e da mãe foi obtida pelo relato do entrevistado. Sobre a utilização dos serviços de saúde, consideraramse as consultas médicas realizadas no período referente aos 12 meses anteriores à entrevista.

As entrevistas foram realizadas por 30 entrevistadoras do sexo feminino com, pelo menos, o segundo grau completo de escolaridade, selecionadas mediante entrevista e prova escrita. O treinamento teve duração de 40 horas, abordando técnicas de entrevista, aplicação do questionário e utilização do monitor de pulso para medida da pressão arterial. Para a segunda visita, foram selecionadas e treinadas duas técnicas em enfermagem.

O estudo-piloto foi realizado em área não sorteada para integrar a amostra, com todas as entrevistadoras que realizaram o treinamento, sob a supervisão dos mestrandos. Com o estudo piloto, foi possível detectar problemas no questionário, calcular o tempo da entrevista e avaliar o desempenho das entrevistadoras.

O controle de qualidade da primeira visita foi realizado pelo supervisor do estudo, em amostra aleatória de $10 \%$ das entrevistas, valendo-se da reaplicação de algumas perguntas do questionário. Utilizou-se o índice kappa para avaliar a concordância entre a resposta obtida pelo entrevistador e pela coordenadora do estudo, a qual foi responsável pelo controle de qualidade. O con- 
Figura 1

Fluxograma do estudo. Pelotas, Rio Grande do Sul, Brasil, 2007.

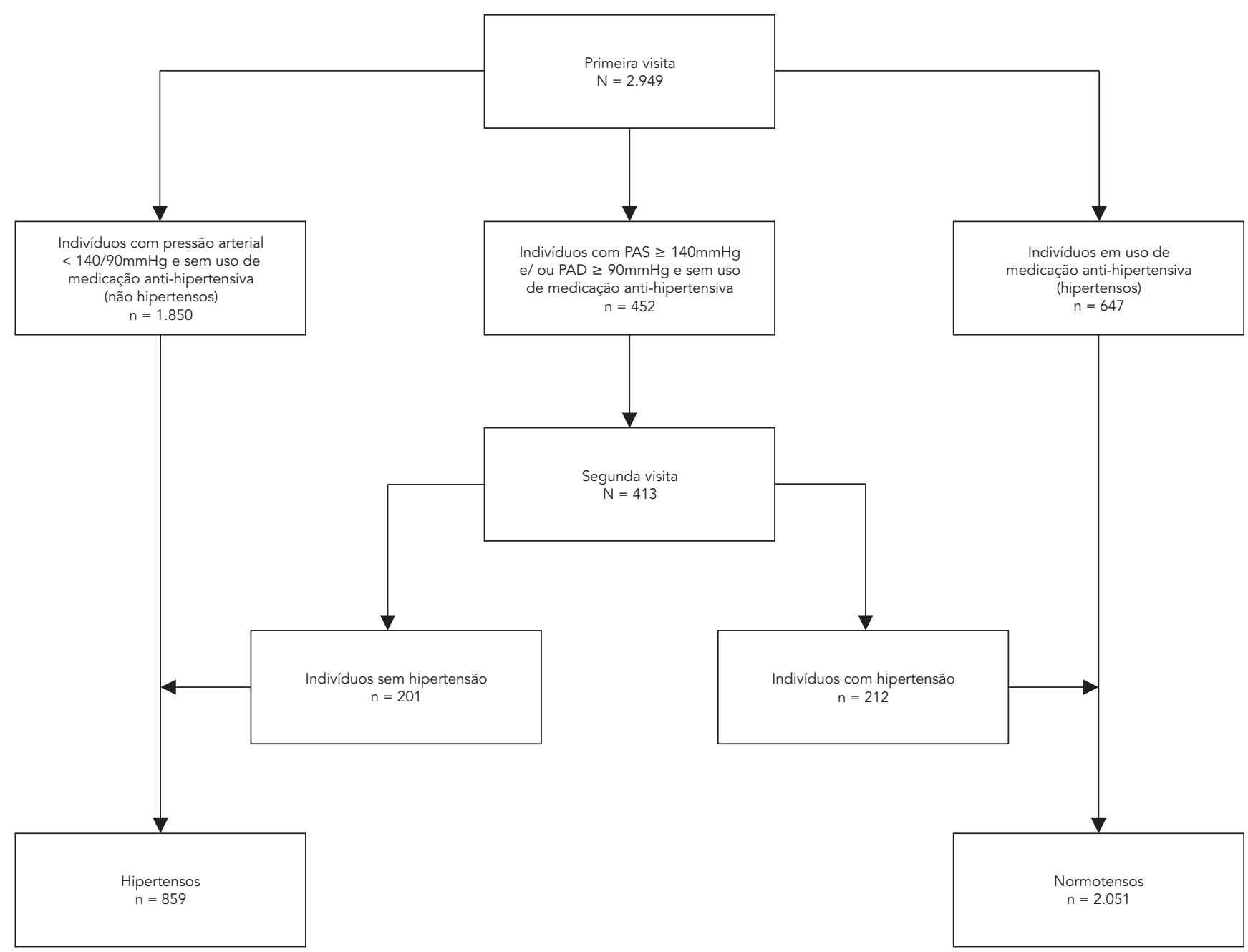

PAS: pressão arterial sistólica; PAD: pressão arterial diastólica.

trole de qualidade da segunda visita foi feito por telefone com $10 \%$ dos indivíduos selecionados por meio de sorteio. A supervisora do trabalho perguntava sobre a visita da segunda entrevistadora e certificava-se de que a mesma havia verificado a pressão arterial do participante.

\section{Análise dos dados}

Os dados das duas visitas foram duplamente digitados, comparados e corrigidos através do programa Epi Info versão 6.04 (Centers for Disease Control and Prevention, Atlanta, Estados Uni- dos), e a análise foi realizada através do programa Stata versão 9.0 (Stata Corp., College Station, Estados Unidos). As prevalências de hipertensão arterial auto-referida e medidas (padrão-ouro) foram calculadas e comparadas mediante o teste qui-quadrado 20 .

Foram calculadas as seguintes propriedades do auto-relato de hipertensão: sensibilidade, especificidade e valores preditivos positivo e negativo. A sensibilidade forneceu a proporção de indivíduos hipertensos, conforme o padrão-ouro, que relataram ter hipertensão; a especificidade, a proporção de normotensos que relataram não 
ter hipertensão. O valor preditivo positivo (VPP) foi obtido pela proporção de verdadeiros hipertensos entre os que relataram ter hipertensão. O valor preditivo negativo (VPN) foi dado pela proporção de verdadeiros normotensos entre aqueles que se declararam não hipertensos 21 . Foram calculados intervalos de $95 \%$ de confiança (IC95\%) para cada um desses parâmetros.

As propriedades do auto-relato também foram calculadas para cada estrato das variáveis independentes. Para explorar as características individuais que se associavam independentemente com a acurácia e o VPP do auto-relato, realizou-se regressão de Poisson com variância robusta. Na regressão, todas as variáveis foram levadas ao modelo inicial e retiradas por seleção retrógrada, independentemente de estarem associadas à acurácia ou ao VPP em análise bivariada. O critério para uma variável ser mantida no modelo foi estar associada ao desfecho com $\mathrm{p}<0,05$.

\section{Aspectos éticos}

O projeto de pesquisa deste estudo foi submetido ao Comitê de Ética em Pesquisa da Faculdade de Medicina da UFPEL, sendo aprovado através do ofício $\mathrm{n}^{\circ}$. 088/07. A entrevista somente foi realizada após o participante assinar o Termo de Consentimento Informado. Tanto na primeira quanto na segunda visita, todos os participantes da pesquisa receberam um cartão contendo o valor da última medida da pressão arterial. Aqueles com pressão arterial igual ou superior a 140/90mmHg foram aconselhados a realizar consulta médica.

\section{Resultados}

Foram localizados 1.534 domicílios. Destes, 1.522 eram residenciais e estavam habitados, sendo, portanto, elegíveis para o estudo. De um total de 3.180 indivíduos com idade igual ou superior a 20 anos, foram entrevistados 2.986 , o que representa uma taxa de respondentes de 94,9\%. A Figura 1 apresenta o fluxograma do estudo com o número de indivíduos localizados em cada uma das visitas e prevalência de hipertensos e normotensos nos diferentes momentos de abordagem dos participantes. Na primeira visita, 98,8\% (2.949) da amostra tiveram a pressão arterial aferida. Deste total, 21,9\% (647) estavam em uso de medicação anti-hipertensiva e 15,3\% (452) tiveram a PAS e/ ou a $\mathrm{PAD} \geq 140 / 90 \mathrm{mmHg}$. Estes últimos receberam a segunda visita, cuja taxa de participação foi de $91,4 \%$ (413), sendo $48,7 \%$ (212) classificados como hipertensos. No controle de qualidade, o índice kappa de concordância mostrou que a repetibilidade da resposta à pergunta "Algum médico já lhe disse que o Sr.(a) tem pressão alta?”, testada em média $18( \pm 11)$ dias após entrevista, foi de 0,8 .

A Tabela 1 descreve as características da população estudada. Mais da metade da amostra foi composta por mulheres (56,7\%), cerca de dois terços dos participantes tinham entre 20 e 49 anos de idade e 79,3\% classificaram-se como brancos. Quanto à escolaridade, cerca de $60 \%$ tinham de 5 a 12 anos de educação formal, sendo a média de $8( \pm 4,7)$ anos. A mediana de renda familiar foi de R\$1.250,00 mensais. Havia história de hipertensão arterial do pai e da mãe, respectivamente, para $29,5 \%$ e $46,7 \%$ dos entrevistados. Pouco mais da metade dos indivíduos estudados nunca havia fumado (52,6\%), 51,7\% foram classificados pelo IPAQ como sedentários e mais de três quartos $(78,1 \%)$ haviam consultado com médico pelo menos uma vez nos últimos 12 meses.

A Tabela 1 mostra ainda a prevalência de hipertensão auto-referida e conforme o padrãoouro, segundo características sócio-demográficas, familiares, comportamentais e de utilização de consultas médicas. A prevalência de hipertensão arterial auto-referida de 33,6\% foi superior à obtida segundo o padrão-ouro ( $\mathrm{p}<0,001)$.

Cerca de $40 \%$ das mulheres disseram-se hipertensas, enquanto o padrão-ouro mostrou que $31 \%$ eram, de fato, hipertensas. A prevalência de hipertensão arterial aumentou progressivamente com a idade nos dois parâmetros utilizados. A auto-referida passou de 11,3\% entre indivíduos com 20 a 29 anos, para $61,9 \%$ entre aqueles com 60 anos ou mais. No caso da prevalência conforme o padrão-ouro, entre essas faixas etárias, passou de $4,6 \%$ para $65,4 \%$. Indivíduos com baixa escolaridade tiveram maior prevalência de hipertensão auto-referida (47,3\%) e de hipertensão pelo padrão-ouro (45\%) do que os com 13 anos de educação formal (respectivamente $25,6 \%$ e 21,8\%).

A prevalência de hipertensão auto-referida foi maior que a obtida pelo padrão-ouro para todas as características estudadas. No entanto, as diferenças não foram estatisticamente significativas para o sexo masculino, para aqueles com idade igual ou superior a 50 anos, com cor da pele parda, nos extremos de escolaridade, para aqueles que diziam que o pai não tinha hipertensão e para os que não haviam consultado médico nos últimos 12 meses (Tabela 1).

A sensibilidade e o VPP para a hipertensão auto-referida foram, respectivamente, $84,3 \% \mathrm{e}$ $73,9 \%$. A especificidade e o valor preditivo negativo foram $87,5 \%$ e $93 \%$, respectivamente. $\mathrm{Na}$ Tabela 2, estão descritas as propriedades do auto-relato de hipertensão, conforme as carac- 
Tabela 1

Características demográficas, sócio-econômicas, comportamentais, de história familiar de hipertensão, de utilização de consulta médica e de prevalência da hipertensão auto-referida e conforme o padrão-ouro de amostra representativa da população de Pelotas, Rio Grande do Sul, Brasil, 2007.

\begin{tabular}{|c|c|c|c|c|}
\hline \multirow[t]{2}{*}{ Características } & \multirow[t]{2}{*}{ n (\%) } & \multicolumn{2}{|c|}{ Hipertensão arterial sistêmica } & \multirow[t]{2}{*}{ Valor de $p$ * } \\
\hline & & $\begin{array}{c}\text { Auto-referida } \\
\text { n (\%) }\end{array}$ & $\begin{array}{l}\text { Padrão-ouro } \\
\text { n (\%) }\end{array}$ & \\
\hline \multicolumn{5}{|l|}{ Sexo } \\
\hline Masculino & $1.277(43,3)$ & $1.274(26,8)$ & $1.255(27,7)$ & 0,30 \\
\hline Feminino & $1.672(56,7)$ & $1.671(38,8)$ & $1.655(30,9)$ & $<0,001$ \\
\hline \multicolumn{5}{|l|}{ Idade (anos) } \\
\hline $20-29$ & $701(23,8)$ & $701(11,3)$ & $694(4,6)$ & $<0,001$ \\
\hline $30-39$ & $544(18,4)$ & $544(20,6)$ & $540(12,2)$ & $<0,001$ \\
\hline $40-49$ & $594(20,1)$ & $592(32,4)$ & $584(25,2)$ & $<0,001$ \\
\hline $50-59$ & $517(17,5)$ & $517(46,6)$ & $509(45,8)$ & 0,79 \\
\hline 60 ou mais & $593(20,1)$ & $591(61,9)$ & $583(65,4)$ & 0,22 \\
\hline \multicolumn{5}{|c|}{ Cor da pele auto-referida } \\
\hline Branca & $2.334(79,3)$ & $2.330(32,5)$ & $2.307(28,9)$ & 0,008 \\
\hline Preta & $400(13,6)$ & $400(40,3)$ & $391(33,5)$ & 0,04 \\
\hline Parda & $209(7,1)$ & $209(32,1)$ & $206(28,6)$ & 0,45 \\
\hline \multicolumn{5}{|l|}{ Escolaridade (anos) } \\
\hline $0-4$ & $696(23,6)$ & $694(47,3)$ & $682(45,0)$ & 0,40 \\
\hline $5-8$ & $928(31,5)$ & $926(34,8)$ & $916(29,8)$ & 0,02 \\
\hline $9-12$ & $827(28,1)$ & $827(25,6)$ & $820(20,9)$ & 0,02 \\
\hline 13 ou mais & $496(16,8)$ & $496(25,6)$ & $490(21,8)$ & 0,16 \\
\hline \multicolumn{5}{|c|}{ Renda familiar (quartis) ** } \\
\hline Primeiro (menor) & $723(26,5)$ & $722(33,9)$ & $710(29,3)$ & 0,06 \\
\hline Segundo & $655(24,0)$ & $655(32,7)$ & $647(29,4)$ & 0,20 \\
\hline Terceiro & $688(25,2)$ & $686(36,4)$ & $684(31,1)$ & 0,04 \\
\hline Quarto (maior) & $661(24,2)$ & $661(28,9)$ & $653(25,9)$ & 0,22 \\
\hline \multicolumn{5}{|c|}{ História familiar de hipertensão arterial } \\
\hline \multicolumn{5}{|c|}{ Pai } \\
\hline Não & $1.611(54,6)$ & $1.610(27,0)$ & $1.589(24,5)$ & 0,10 \\
\hline $\operatorname{Sim}$ & $869(29,5)$ & $867(41,8)$ & $860(33,3)$ & $<0,001$ \\
\hline Ignorado & $469(15,9)$ & $468(41,2)$ & $461(39,9)$ & 0,68 \\
\hline \multicolumn{5}{|l|}{ Mãe } \\
\hline Não & $1.262(42,8)$ & $1.261(25,5)$ & $1.247(21,1)$ & 0,007 \\
\hline Sim & $1.378(46,7)$ & $1.377(38,4)$ & $1.360(33,8)$ & 0,01 \\
\hline Ignorado & $309(10,5)$ & $307(45,3)$ & $303(45,2)$ & 0,99 \\
\hline \multicolumn{5}{|l|}{ Tabagismo } \\
\hline Nunca fumou & $1.550(52,6)$ & $1.549(33,1)$ & $1.530(29,5)$ & 0,03 \\
\hline Fumante atual & $754(25,6)$ & $753(27,2)$ & $742(22,9)$ & 0,05 \\
\hline Ex-tabagista & $645(21,9)$ & $643(42,5)$ & $638(37,3)$ & 0,06 \\
\hline \multicolumn{5}{|l|}{ Atividade física ** } \\
\hline Ativo & $1.415(48,3)$ & $1.413(30,4)$ & $1.398(26,1)$ & 0,01 \\
\hline Sedentário & $1.517(51,7)$ & $1.515(36,6)$ & $1.496(32,6)$ & 0,02 \\
\hline \multicolumn{5}{|c|}{ Consulta médica nos últimos 12 meses ** } \\
\hline Não & $608(21,9)$ & $606(18,7)$ & $604(18,9)$ & 0,92 \\
\hline Sim & $2.173(78,1)$ & $2.171(35,7)$ & $2.142(29,5)$ & $<0,001$ \\
\hline Total & $2.949(100,0)$ & $2.945(33,6) * \star$ & $2.910(29,5) * \star$ & $<0,001$ \\
\hline
\end{tabular}

* Teste qui-quadrado;

** Valores ignorados para: escolaridade (2), classe social (29), renda familiar (219), atividade física (16), consulta médica (166), hipertensão arterial sistêmica auto-referida (4) e hipertensão medida (17). 
terísticas da amostra. Não houve diferença importante na sensibilidade para a maioria das variáveis investigadas, como demonstra a sobreposição dos intervalos de confiança, exceto para sexo, idade, tabagismo e consulta a médico nos últimos 12 meses. A sensibilidade foi maior entre as mulheres $(92,2 \%)$ do que entre os homens $(72,7 \%)$, entre os ex-tabagistas $(88,7 \%)$, quando comparados aos fumantes atuais $(75,3 \%)$, e entre os que realizaram consulta médica nos últimos 12 meses (88,3\% contra 63,2\%). A sensibilidade foi diferente nos extremos de idade, sendo de 56,2\% para indivíduos entre 20-29 anos e superior a $80 \%$ entre aqueles com 40 anos ou mais. Para todas as categorias de cor da pele, escolaridade, renda familiar, história familiar de hipertensão e nível de atividade física, a sensibilidade foi de pelo menos $80 \%$ (Tabela 2 ).

Com a especificidade, os resultados foram inversos, sendo esta maior entre os homens $(91 \%$ contra $84,7 \%$ das mulheres) e entre os que não consultaram com médico nos últimos 12 meses (91,6\% contra $86,2 \%)$. Tal como a sensibilidade, para as demais variáveis, a especificidade foi superior a $80 \%$ em todos os estratos.

Entre todas as categorias das variáveis investigadas, houve diferença no VPP conforme o grupo etário e hábito de fumar. Os indivíduos com 60 anos ou mais tiveram o maior VPP (92,5\%), em relação àqueles nas demais faixas etárias, o mesmo se dando com os ex-tabagistas $(77,9 \%)$, quando comparado aos tabagistas atuais $(63,4 \%)$.

Mais de $80 \%$ dos indivíduos entrevistados responderam corretamente quanto a sua condição de apresentar hipertensão arterial (acurácia de 86,5\%; IC95\%: 85,1-87,8\%). A análise de regressão de Poisson mostrou que a probabilidade de resposta correta, tanto na presença, como na ausência de hipertensão, esteve associada somente com a escolaridade. Ressalte-se que indivíduos com alta escolaridade (13 anos ou mais) apresentaram uma probabilidade $8 \%$ maior de responder corretamente do que os com menos de cinco anos de educação formal $(\mathrm{RP}=1,08$; IC95\%: 1,03-1,12).

Com relação ao VPP, a análise de regressão de Poisson mostrou que as variáveis sexo, idade e consulta médica nos últimos 12 meses estavam independentemente associadas à correta presença de hipertensão arterial, quando o indivíduo se dizia hipertenso (VPP). A RP para o VPP foi de 1,24 (IC95\%: 1,08-1,43) para as mulheres em comparação aos homens tomados como referência. A associação com a idade foi direta e linear, com RP de 3,72, 8,46, 15,09 e 21,92, respectivamente, para as faixas etárias de 30-39, 40-49, 50-59 e 60 anos ou mais. Entre os indivíduos que consultaram nos últimos 12 meses, a probabili- dade de se referirem hipertensos quando, de fato, o eram foi $80 \%$ maior do que entre os que não consultaram $(\mathrm{RP}=1,80$; IC95\%: 1,44-2,27).

\section{Discussão}

Na prática clínica, o método ideal para avaliar a presença de hipertensão em cada paciente é a avaliação da média de várias medidas da pressão arterial em dias diferentes 18. Entretanto, para a realização de grandes inquéritos populacionais, torna-se logisticamente difícil e oneroso realizar mais de uma visita aos participantes. Vários estudos utilizam apenas uma medida da pressão arterial 4,5,6,7,8,11,13,22,23,24, ou o auto-relato 6,9,12,25,26 para determinar a prevalência de hipertensão arterial.

Este estudo mostrou que o auto-relato é uma estratégia válida para identificar a prevalência de hipertensão em estudos de base populacional, tendo uma sensibilidade de $84,3 \%$, especificidade de $87,5 \%$ e repetibilidade de $80 \%$. Apenas um estudo brasileiro de base populacional com o objetivo de medir a validade do auto-relato da hipertensão arterial foi localizado na literatura 16 . Nessa investigação, realizada em Bambuí, Minas Gerais, com 970 indivíduos de 18 anos ou mais de idade, o padrão-ouro foi definido com base na média de duas medidas da pressão arterial tomadas em uma mesma visita e/ou no uso em curso de medicamentos anti-hipertensivos. A sensibilidade e especificidade do auto-relato foram, respectivamente, $72,1 \%$ (IC95\%: 69,3-75,0\%) e 86,4\% (IC95\%: 84,3-88,6\%). Os intervalos de confiança indicam que a sensibilidade foi maior entre os participantes em Pelotas, e que a especificidade foi semelhante nas duas cidades.

Trabalhos conduzidos nos Estados Unidos mostraram sensibilidade variada, entre $71 \%$ (semelhante à da investigação de Bambuí) e $83 \%$ (semelhante à do estudo de Pelotas) e alta especificidade (80\%), como os dois estudos brasileiros $27,28,29$. Um estudo espanhol identificou sensibilidade de $63,5 \%$ e especificidade de $91,4 \% 30$.

Além de fatores contextuais e de estilo de vida, diferentes definições de padrão-ouro e diferentes instrumentos de mensuração da pressão arterial podem ter contribuído com a variação da sensibilidade encontrada na literatura. Alguns autores compararam o auto-relato aos registros médicos 29,30, enquanto outros aferiram a pressão arterial em uma 16 ou mais ocasiões 27 . A pressão arterial foi aferida com esfigmomanômetros aneróides no estudo de Lima-Costa et al. 16 e com esfigmomanômetros de mercúrio no de Vargas et al. 27. O uso de monitores de pulso é recomendado pela Sociedade Brasileira de 
Tabela 2

Propriedades do auto-relato de hipertensão arterial, conforme características sócio-demográficas, familiares, comportamentais e de utilização de consulta médica. Pelotas, Rio Grande do Sul, Brasil, 2007.

\begin{tabular}{|c|c|c|c|c|}
\hline Características & $\begin{array}{l}\text { Sensibilidade } \\
\text { (\%) IC95\% }\end{array}$ & $\begin{array}{l}\text { Especificidade } \\
\text { (\%) IC } 95 \%\end{array}$ & $\begin{array}{c}\text { Valor preditivo } \\
\text { positivo } \\
\text { (\%) IC95\% }\end{array}$ & $\begin{array}{c}\text { Valor preditivo } \\
\text { negativo } \\
\text { (\%) IC95\% }\end{array}$ \\
\hline \multicolumn{5}{|l|}{ Sexo } \\
\hline Masculino & $72,7(67,7-77,3)$ & $91,0(89,0-92,8)$ & $75,8(70,8-80,3)$ & $89,7(87,5-91,6)$ \\
\hline Feminino & $92,2(89,5-4,3)$ & $84,7(82,5-86,7)$ & $72,9(69,3-76,3)$ & $96,0(94,6-97,2)$ \\
\hline \multicolumn{5}{|l|}{ Idade (anos) } \\
\hline $20-29$ & $56,2(37,6-73,6)$ & $90,8(88,3-92,9)$ & $22,8(14,1-33,6)$ & $97,7(96,2-98,8)$ \\
\hline $30-39$ & $78,8(67,0-87,9)$ & $87,3(84,0-90,2)$ & $46,4(37,0-56,1)$ & $96,7(94,6-98,2)$ \\
\hline $40-49$ & $83,0(75,8-88,6)$ & $84,6(80,9-87,9)$ & $64,5(57,3-71,4)$ & $93,6(90,8-95,8)$ \\
\hline $50-59$ & $84,5(79,3-88,9)$ & $85,1(80,4-89,1)$ & $82,8(77,4-87,4)$ & $86,7(82,1-90,5)$ \\
\hline 60 ou mais & $87,9(84,0-91,0)$ & $86,5(81,0-90,9)$ & $92,5(89,3-95,0)$ & $79,0(73,0-84,2)$ \\
\hline \multicolumn{5}{|l|}{ Cor da pele auto-referida } \\
\hline Branca & $84,1(81,1-86,8)$ & $88,3(86,7-89,9)$ & $74,6(71,3-77,5)$ & $93,2(91,8-94,4)$ \\
\hline Preta & $86,3(79,2-91,7)$ & $82,3(77,1-86,8)$ & $71,1(63,4-78,0)$ & $92,2(88,0-95,3)$ \\
\hline Parda & $81,4(69,1-90,3)$ & $87,8(81,3-92,6)$ & $72,7(60,4-83,0)$ & $92,1(86,4-96,0)$ \\
\hline \multicolumn{5}{|l|}{ Escolaridade (anos) } \\
\hline $0-4$ & $86,3(82,0-90,0)$ & $85,0(81,0-88,5)$ & $82,6(78,0-86,5)$ & $88,3(84,5-91,4)$ \\
\hline $5-8$ & $81,7(76,6-86,1)$ & $85,0(82,0-87,7)$ & $69,9(64,5-74,9)$ & $91,6(89,1-93,7)$ \\
\hline $9-12$ & $80,1(73,2-85,8)$ & $88,4(85,7-90,8)$ & $64,6(57,8-70,1)$ & $94,4(92,3-96,1)$ \\
\hline 13 ou mais & $91,6(84,6-96,1)$ & $92,4(89,3-94,9)$ & $77,2(68,9-84,1)$ & $97,5(95,4-98,9)$ \\
\hline \multicolumn{5}{|l|}{ Renda familiar (quartis) } \\
\hline Primeiro (menor) & $82,2(76,3-87,2)$ & $86,0(82,7-88,9)$ & $70,9(64,8-76,6)$ & $92,1(89,3-94,4)$ \\
\hline Segundo & $83,2(77,1-88,2)$ & $88,2(84,9-91,0)$ & $74,5(68,1-80,3)$ & $92,6(89,8-94,9)$ \\
\hline Terceiro & $83,6(77,9-88,3)$ & $85,1(81,5-88,2)$ & $71,8(65,7-77,3)$ & $91,9(89,0-94,3)$ \\
\hline Quarto (maior) & $87,6(81,6-92,1)$ & $91,3(88,5-93,7)$ & $77,9(71,3-83,6)$ & $95,5(93,2-97,2)$ \\
\hline \multicolumn{5}{|l|}{ Pai hipertenso } \\
\hline Não & $81,2(77,0-85,0)$ & $90,6(88,8-92,2)$ & $73,3(69,2-77,8)$ & $93,7(92,1-95,0)$ \\
\hline Sim & $88,8(84,6-92,2)$ & $81,6(78,2-84,7)$ & $70,7(65,7-75,4)$ & $93,6(91,1-95,6)$ \\
\hline Ignorado & $83,7(77,6-88,7)$ & $86,2(81,6-90,1)$ & $80,2(73,9-85,6)$ & $88,8(84,4-92,3)$ \\
\hline \multicolumn{5}{|l|}{ Mãe hipertensa } \\
\hline Não & $81,7(76,5-86,2)$ & $89,5(87,4-91,4)$ & $67,6(62,2-72,7)$ & $94,8(93,2-96,2)$ \\
\hline $\operatorname{Sim}$ & $85,2(81,6-88,3)$ & $85,2(82,7-87,5)$ & $74,6(70,7-78,3)$ & $91,9(89,8-93,6)$ \\
\hline Ignorado & $86,1(79,2-91,4)$ & $87,8(81,8-92,4)$ & $85,5(78,5-90,9)$ & $88,3(82,4-92,8)$ \\
\hline \multicolumn{5}{|l|}{ Tabagismo } \\
\hline Nunca fumou & $85,4(81,8-88,5)$ & $88,7(86,6-90,5)$ & $75,9(72,0-79,6)$ & $93,5(91,9-95,0)$ \\
\hline Fumante atual & $75,3(68,1-81,6)$ & $87,0(84,0-89,7)$ & $63,4(56,3-70,0)$ & $92,2(89,6-94,3)$ \\
\hline Ex-tabagista & $88,7(83,9-92,4)$ & $84,9(81,0-88,3)$ & $77,9(72,4-82,7)$ & $92,6(89,4-95,1)$ \\
\hline \multicolumn{5}{|l|}{ Atividade física } \\
\hline Ativo & $79,8(75,0-84,1)$ & $87,0(84,6-89,2)$ & $69,1(64,1-73,8)$ & $92,2(90,2-94,0)$ \\
\hline Sedentário & $87,2(84,1-89,9)$ & $87,8(85,8-89,7)$ & $76,7(73,2-80,1)$ & $93,7(92,1-95,1)$ \\
\hline \multicolumn{5}{|l|}{ Consultou com médico nos últimos 12 meses } \\
\hline Sim & $88,3(85,5-90,7)$ & $86,2(84,4-87,9)$ & $72,9(69,5-76,0)$ & $94,6(93,3-95,8)$ \\
\hline Não & $63,2(53,6-72,0)$ & $91,6(88,8-93,6)$ & $63,7(54,1-72,6)$ & $91,4(88,6-93,7)$ \\
\hline Hipertensão arterial sistêmica auto-referida & $84,3(81,7-86,7)$ & $87,5(86,0-88,9)$ & $73,9(71,0-76,6)$ & $93,0(91,8-94,1)$ \\
\hline
\end{tabular}


Cardiologia. Seu uso é mais simples e previne o erro de leituras dependentes da adequada identificação dos sons de korotkoff, necessária com os instrumentos aneróides e de mercúrio. O monitor utilizado neste estudo está aprovado pelos seguintes protocolos: British Hypertension Society e Association for the Advancement of Medical Instrumentation (British Hypertension Society. http://www.bhsoc.org/bp_monitors/automa tic_wrist.stm, acessado em 28/Set/2008).

No atual estudo, o valor preditivo do autorelato de hipertensão foi de $74 \%$, indicando que, aproximadamente, três em cada quatro indivíduos que se declararam hipertensos de fato o eram. Tal VPP é decorrente da alta especificidade do auto-relato e da alta prevalência de hipertensão arterial na população estudada. O estudo de Bambuí 16 e o National Health and Nutrition Examination Survey III (NHANES III) 27 encontraram valores preditivos semelhantes, com prevalências de hipertensão, respectivamente, de 23\% e 27\%.

Com relação aos subgrupos analisados, a sensibilidade do auto-relato entre as mulheres pelotenses foi maior do que entre as de Bambuí (92\% contra $79 \%)$. As diferenças encontradas na sensibilidade e especificidade, entre os sexos, e na sensibilidade, entre extremos de idade e condição de tabagismo, podem ter explicação no acesso aos serviços de saúde. Estudo realizado na cidade de Pelotas mostrou que as mulheres consultam mais do que os homens, logo elas têm maior oportunidade de receber o diagnóstico de hipertensão ${ }^{31}$. Com relação à idade, a sensibilidade maior a partir dos 50 anos pode dever-se ao crescimento da prevalência de hipertensão com o aumentar da idade. A presença de outras enfermidades, cujas prevalências também aumentam com a idade, resultando em consulta médica, aumentaria as oportunidades de diagnóstico de hipertensão. O mesmo ocorre com ex-tabagistas, uma vez que a modificação deste comportamento pode ter sido influenciada pela instalação de doenças que os levaram a procurar os serviços de saúde.

A prevalência de hipertensão arterial autoreferida de $34 \%$, encontrada no presente estudo, foi maior do que a de 23\% (IC95\%: 22,0-25,0), observada na mesma cidade, em 2003 32, e a de $27 \%$ (IC95\%: 24,4-30,1), registrada em Bambuí. As possíveis razões para essas diferenças incluem a não padronização, nos questionários utilizados, das perguntas sobre hipertensão, mudanças nos critérios clínicos de diagnóstico de hipertensão, oportunidade de o indivíduo ter recebido de um profissional o diagnóstico e mudança real na prevalência do desfecho.

$\mathrm{O}$ primeiro caso pode estar relacionado à diferença encontrada em relação a Bambuí, uma vez que naquele estudo a pergunta não se restringia a diagnóstico médico de hipertensão (" $A l$ gum médico ou profissional de saúde já disse que você tinha pressão alta ou hipertensão arterial?"). Se tudo o mais fosse semelhante ao atual estudo, exceto a pergunta sobre hipertensão, esta maior flexibilidade teria resultado em maior prevalência de hipertensão auto-referida em Bambuí. Por sua vez, o estudo de Pelotas de 200331 investigou hipertensão auto-referida utilizando a seguinte pergunta: "O médico já lhe disse que o (a) Sr. (a) tem algumas destas doenças?" e a segunda opção de uma lista de seis doenças era: "pressão alta ou hipertensão".

A segunda razão (mudança nos critérios clínicos) não parece ter ocorrido, uma vez que o estudo de Bambuí adotou o mesmo ponto de corte para o padrão-ouro (140/90mmHg), só que em apenas uma medida. A oportunidade de o indivíduo ter recebido o diagnóstico de hipertensão, por sua vez, depende do acesso e utilização dos serviços de saúde e do critério diagnóstico em voga na clínica médica. A esse respeito, no atual estudo, $78 \%$ dos participantes utilizaram serviços médicos ambulatoriais no último ano e, em Bambuí, 77\% haviam consultado há menos de dois anos. Dados de Pelotas mostram que a aferição da pressão arterial de adultos na rede básica ocorre em 55,5\% das consultas, independente da queixa principal (Capilheira MF, Santos IS. Comunicação pessoal; 2009).

Quanto à possível mudança na prevalência do desfecho, a reanálise de um estudo de base populacional de Pelotas, realizado em 1999 13, com a pressão arterial medida uma única vez, mostrou que, caso o critério diagnóstico fosse o atualmente em voga, a prevalência de hipertensão seria de 37,2\% (IC95\%: 35,1-39,4). Se o atual estudo adotasse esse padrão-ouro, a prevalência de hipertensão seria muito semelhante $(37,3 \%$; IC95\%: 35,5-39,0), com sensibilidade e especificidade do auto-relato de 71,3\% (IC95\%: 68,5-73,9) e 88,8\% (IC95\%: 87,3-90,2), respectivamente. Tal achado indica que a prevalência real de hipertensão arterial não aumentou nos últimos oito anos.

Metodologicamente, uma das limitações deste estudo é o tempo de seis semanas decorridas entre a primeira e a segunda medida, esta tirada dos indivíduos com níveis pressóricos elevados na primeira visita. Nesse período, três pessoas haviam iniciado o uso de medicação anti-hipertensiva, e duas permaneciam com a pressão elevada. No entanto, esse fato não resultou em erro de classificação do desfecho, sendo as três classificadas como hipertensas devido ao uso de anti-hipertensivos. Modificações no estilo de vida ao longo dessas seis semanas também podem 
ter ocorrido, entretanto há evidências de que o efeito do estilo de vida saudável sobre redução dos níveis pressóricos torna-se evidente, em geral, após transcorrido um período de pelo menos seis meses 32 .

Aspectos positivos deste estudo incluem seu caráter populacional e o reduzido número de perdas e recusas. Quanto ao primeiro, o número de indivíduos estudados e a estratégia de amostragem resultaram em uma amostra cuja distribuição em termos de idade, sexo e renda familiar média é semelhante às das estimativas censitárias do IBGE para Pelotas, no ano de 2007 (Instituto Brasileiro de Geografia e Estatística. http:/ / www.ibge.gov.br/cidadesat/topwindow.htm?1, acessado em 01/Set/2007). Quanto às perdas e recusas, o estudo contou com uma alta taxa de participação nas primeira e segunda visitas, reduzindo o risco de viés de seleção. A sensibilidade observada do auto-relato $(84,3 \%)$, superior à estimada por ocasião do planejamento do estudo (70\%), permitiu que este parâmetro fosse estimado com um erro de 2,5 pontos percentuais, dados os 859 hipertensos da amostra.

O Brasil é um país que vem acumulando experiência em inquéritos populacionais para medir a prevalência auto-referida de hipertensão. Em 1998, o suplemento de saúde da Pesquisa Nacional por Amostra de Domicílios (PNAD) estimou em $20 \%$ a prevalência na população adul- ta 6. Entre 2000 e 2003, um inquérito domiciliar em 15 capitais brasileiras e no Distrito Federal, conduzido pelo Instituto Nacional de Câncer (INCA), encontrou prevalências variando de 7,4\% a $15,7 \%$ nas pessoas com $25-39$ anos de idade, de $26 \%$ a $36,4 \%$ naquelas entre $40-59$ anos e de $39 \%$ a $59 \%$ nos idosos 9. Mais recentemente, o estudo implantado pelo sistema VIGITEL (Vigilância dos Fatores de Risco e Proteção para Doenças Crônicas por Inquérito Telefônico) nas capitais brasileiras, realizado em 2006, demonstrou que a hipertensão auto-referida variou de $15,1 \%$ a $23,9 \%$ entre adultos com idade superior ou igual a 18 anos 25 . O presente estudo mostrou que a taxa de respostas falso-positivas foi da ordem de $15 \%$, aproximadamente. Futuras replicações dos inquéritos supracitados deverão considerar a tendência da prevalência referida, descontada a taxa esperada de respostas falso-positivas.

Não obstante as diferenças regionais de escolaridade, sexo, idade e acesso a serviços de saúde, o atual estudo demonstra que o auto-relato de hipertensão é válido e pode ser usado em nosso meio para monitorar mudanças na prevalência desse importante fator de risco para doenças crônicas não transmissíveis. Contudo, a padronização da pergunta nos diferentes inquéritos é essencial para que os resultados possam ser comparáveis.

\section{Resumo}

Com o objetivo de investigar a validade do auto-relato de hipertensão arterial, realizou-se estudo transversal, de base populacional, com indivíduos de 20 anos ou mais de idade, residentes em Pelotas, Rio Grande do Sul, Brasil, selecionados por amostragem probabilística em dois estágios. A pressão arterial foi medida duas vezes (cinco minutos de intervalo) em 2.949 participantes visitados em casa. Aqueles com pressão sistóli$c a \geq 140 \mathrm{mmHg}$ elou diastólica $\geq 90 \mathrm{mmHg}$ foram revisitados, e a pressão medida mais duas vezes. Conforme padrão-ouro, hipertensão foi definida pela média das pressões na segunda visita ou uso de medicação anti- hipertensiva. O auto-relato foi obtido por meio da pergunta: "Algum médico disse que o(a) Sr.(a) tem pressão alta?". A prevalência auto-referida foi $33,6 \%$, e a medida, 29,5\%. A sensibilidade foi 84,3\% (IC95\%: 81,786,7), especificidade 87,5\% (IC95\%: 86,0-88,9), valor preditivo positivo 73,9\% (IC95\%: 71,0-76,6) e negativo de 93,0\% (IC95\%: 91,8-94,1). O auto-relato mostrou-se válido para monitoração da prevalência de hipertensão, um dos mais importantes fatores de risco para as doenças crônicas não transmissíveis.

Hipertensão; Doença Crônica; Saúde do Adulto 


\section{Colaboradores}

M. A. D. Chrestani participou da elaboração do projeto, análise de dados e da redação final do artigo. I. S. Santos auxiliou na elaboração do projeto, análise dos dados e redação do artigo. A. M. Matijasevich contribuiu com a análise dos dados e redação do artigo.

\section{Referências}

1. World Health Organization. The World Health Report, 2002: reducing risks, promoting healthy life. Geneva: World Health Organization; 2002.

2. Lawes CMM, Hoorn SV, Rodgers A; for the International Society of Hypertension. Global burden of blood-pressure-related disease, 2001. Lancet 2008; 371:1513-8.

3. Santos IS. Guia metodológico de avaliação e definição de indicadores: doenças crônicas nãotransmissíveis e Rede Carmen. Brasília: Editora do Ministério da Saúde; 2007.

4. Piccini RX, Victora CG. Systemic arterial hypertension in a urban area of southern Brazil: prevalence and risk factors. Rev Saúde Pública 1994; 28:261-7.

5. Fuchs FD, Moreira LB, Moraes RS, Bredemeier M, Cardozo SC. Prevalence of systemic arterial hypertension and associated risk factors in the Porto Alegre metropolitan area. Populational-based study. Arq Bras Cardiol 1994; 63:473-9.

6. Instituto Brasileiro de Geografia e Estatística. Pesquisa Nacional por Amostra de Domicílios (PNAD). Rio de Janeiro: Instituto Brasileiro de Geografia e Estatística; 1998.
7. Klein CH, Silva NAS, Nogueira AR, Bloch KV, Campos LHS. Hipertensão arterial na Ilha do Governador, Rio de Janeiro, Brasil. I. Metodologia. Cad Saúde Pública 1995; 11:187-201.

8. Klein CH, Souza-Silva NA, Nogueira AR, Bloch $\mathrm{KV}$, Campos LHS. Hipertensão arterial na Ilha do Governador, Rio de Janeiro, Brasil. II. Prevalência. Cad Saúde Publica 1995; 11:389-94.

9. Coordenadoria de Prevenção e Vigilância, Instituto Nacional de Câncer. Inquérito domiciliar sobre comportamento de risco e morbidade referida de doenças e agravos não transmissíveis: Brasil, 15 capitais e Distrito Federal. Rio de Janeiro: Instituto Nacional de Câncer; 2004.

10. Onysko J, Maxwell C, Eliasziw M, Zhang JX, Johansen H, Campbell NR. Large increases in hypertension diagnosis and treatment in Canada after a healthcare professional education program. Hypertension 2006; 48:853-60.

11. Lessa I, Magalhães L, Araújo MJ, Almeida Filho N, Aquino E, Oliveira MMC. Hipertensão arterial na população adulta de Salvador (BA) - Brasil. Arq Bras Cardiol 2006; 87:747-56. 
12. Pitsavos C, Milias GA, Panagiotakos DB, Xenaki D, Panagopoulos G, Stefanadis C. Prevalence of selfreported hypertension and its relation to dietary habits, in adults; a nutrition \& health survey in Greece. BMC Public Health 2006; 6:206.

13. Dias-da-Costa J, Barcellos F, Sclowitz M, Sclowitz I, Castanheira M, Olinto M, et al. Prevalência de hipertensão arterial em adultos e fatores associados: um estudo de base populacional urbana em Pelotas, Rio Grande do Sul, Brasil. Arq Bras Cardiol 2007; 88:59-65.

14. Chobanian AV, Bakris GL, Black HR, Cushman WC, Green LA, Izzo Jr. JL, et al. The Seventh report of the Joint National Committee on Prevention, Detection, Evaluation, and Treatment of High Blood Pressure: the JNC 7 report. JAMA 2003; 289:2560-72.

15. Barros AJD, Menezes AMB, Santos IS, Assunção MCF, Gigante D, Fassa AG, et al. O mestrado do Programa de Pós-graduação em Epidemiologia da UFPel baseado em consórcio de pesquisa: uma experiência inovadora. Rev Bras Epidemiol 2008; 11 Suppl 1:133-44.

16. Lima-Costa MF, Peixoto SV, Firmo JO. Validity of self-reported hypertension and its determinants (the Bambuí study). Rev Saúde Pública 2004; 38:637-42.

17. Stefani SD, Barros E. Clínica médica: consulta rápida. 2a Ed. Porto Alegre: Editora Artmed; 2004.

18. The Seventh report of the Joint National Committee on Prevention, Detection, Evaluation, and Treatment of High Blood Pressure. Washington DC: U.S. Department of Health and Human Services; 2004.

19. Craig CL, Marshall AL, Sjöstiom M, Bauman AE, Booth ML, Ainsworth BE, et al. International physical activity questionnaire: 12-country reliability and validity. Med Sci Sports Exerc 2003; 35:138195.

20. Kirkwood B. Essentials of medical statistics. London: Blackwell Scientific Publications; 1988.

21. Fletcher RH, Fletcher SW. Epidemiologia clínica: elementos essenciais. 4a Ed. Porto Alegre: Editora Artmed; 2006.
22. Gus I, Harzheim E, Zaslavsky C, Medina C, Gus M. Prevalence, awareness, and control of systemic arterial hypertension in the state of Rio Grande do Sul. Arq Bras Cardiol 2004; 83:429-33.

23. Beunza JJ, Martinez-Gonzalez MA, Serrano-Martinez M, Alonso A. Incidence of hypertension in a cohort of Spanish university graduates: the SUN study. Rev Esp Cardiol 2006; 59:1331-4.

24. Erem C, Hacihasanoglu A, Kocak M, Deger O, Topbas M. Prevalence of prehypertension and hypertension and associated risk factors among Turkish adults: Trabzon Hypertension Study. J Public Health (Oxf) 2009; 31:45-78.

25. Monteiro CA, Moura EC, Jaime PC, Lucca A, Florindo AA, Figueiredo IC, et al. Surveillance of risk factors for chronic diseases through telephone interviews. Rev Saúde Pública 2005; 39:47-57.

26. Vigitel Brasil 2006. Vigilância de fatores de risco e proteção para doenças crônicas por inquérito telefônico. Brasília: Ministério da Saúde; 2007.

27. Vargas CM, Burt VL, Gillum RF, Pamuk ER. Validity of self-reported hypertension in the National Health and Nutrition Examination Survey III, 1988-1991. Prev Med 1997; 26:678-85.

28. Capilheira MF, Santos IS. Fatores individuais associados à utilização de consultas médicas por adultos. Rev Saúde Pública 2006; 40:436-43.

29. Martin LM, Leff M, Calonge N, Garrett C, Nelson DE. Validation of self-reported chronic conditions and health services in a managed care population. Am J Prev Med 2000; 18:215-8.

30. Tormo MJ, Navarro C, Chirlaque MD, Barber X. Validation of self diagnosis of high blood pressure in a sample of the Spanish EPIC cohort: overall agreement and predictive values. EPIC Group of Spain. J Epidemiol Community Health 2000; 54:221-6.

31. Capilheira M. Prevalência e fatores associados à consulta médica e solicitação de exames complementares: um estudo de base populacional [Dissertação de Mestrado]. Pelotas: Departamento de Medicina Social, Universidade Federal de Pelotas; 2004.

32. Appel LJ, Champagne CM, Harsha DW, Cooper LS, Obarzanek E, Elmer PJ, et al. Effects of comprehensive lifestyle modification on blood pressure control: main results of the PREMIER clinical trial. JAMA 2003; 289:23-30.

Recebido em 24/Jan/2009

Versão final reapresentada em 14/Jul/2009 Aprovado em 01/Set/2009 